\title{
Clinical study of blood pressure variability in aged patients with $\mathrm{H}$ type hypertension
}

\author{
Yongdong Li, Yonghua Gao \\ Department of Cardiology, The Third Affiliated Hospital of Inner Mongolia Medical University, Heart Disease and \\ Macrovascular Disease Institute of Baotou City, Baotou, Inner Mongolia, China
}

Received: June 3, 2017

DOI: $10.14725 /$ dcc.v4n3p10
Accepted: July 15, 2017

URL: http://dx.doi.org/10.14725/dcc.v4n3p10

\begin{abstract}
Objective: To explore characteristics and influencing factors for blood pressure variability (BPV) in aged patients with $\mathrm{H}$ type hypertension.

Methods: A total of 100 patients with essential hypertension, who were treated in our hospital from December 2012 to December 2013, were selected. According to serum level of homocysteine (Hcy), patients were divided into pure hypertension group ( $\mathrm{n}=42, \mathrm{Hcy}<15.4 \mu \mathrm{mol} / \mathrm{L}$ ) and $\mathrm{H}$ type hypertension group. All patients received $24 \mathrm{~h}$ ambulatory blood pressure monitoring (24hABPM), and BPV indexes were recorded and compared between two groups. Correlation among BPV indexes and general data were analyzed, and multi-factor linear regression analysis was used to analyze influencing factors for BPV indexes.

Results: Compared with pure hypertension group, there were significant increases in $24 \mathrm{~h}$ systolic blood pressure standard deviation [24hSSD, $(12.80 \pm 2.23)$ vs. $(16.01 \pm 3.11)$ ], daytime SSD [dSSD, $(11.58 \pm 2.30)$ vs. (13.86 \pm 2.26$)]$ and nighttime SSD [nSSD, $(9.41 \pm 1.64)$ vs. $(11.71 \pm 2.34)]$ in H type hypertension group, $p<.05$ or $p<.01$. Pearson correlation analysis showed that Hcy, total cholesterol (TC) and low-density lipoprotein cholesterol (LDL-C) were significantly positively correlated with $24 \mathrm{hSSD}$, dSSD and nSSD $(r=0.201-0.508, p<.05$ or $p<.01)$. Multivariate linear regression analysis indicated that Hcy and TC were significantly positively correlated with 24hSSD, dSSD and nSSD (B = 0.194-0.546, $p<.05$ or $p<.01)$.

Conclusions: Systolic BPV in aged patients with H type hypertension was significantly higher than that of patients with pure hypertension, their serum Hcy level, TC elevating level were significantly positively correlated with systolic BPV and were major influencing factors for BPV in these patients.
\end{abstract}

Key Words: Hypertension, Homocysteine, Elderly

In recent years, the study points of blood pressure variability (BPV) both at home and abroad mainly focus on the simple essential hypertension population, but there is less research on BPV in elderly patients with $\mathrm{H}$ type hypertension. It is necessary to further clarify the characteristics and main influencing factors of blood pressure fluctuation in elderly patients with $\mathrm{H}$ type hypertension. In this study, patients with hypertension aged 65-82 years were enrolled in this study. Serum homocysteine (Hcy) levels were measured in all subjects. BPV indexes of 24-hour ambulatory blood pressure monitoring (24hABPM) of all subjects were collected to ex- plore the characteristics and influencing factors of BPV in patients with $\mathrm{H}$ type hypertension, so as to provide a new theoretical basis for clinical prevention and treatment of $\mathrm{H}$ type hypertension.

\section{Data and nethods}

\subsection{Research object}

A total of 100 patients with essential hypertension, aged from 65 to 82 years, enrolled or hospitalized in our hospital

\footnotetext{
*Correspondence: Yongdong Li; E-mail: lyd20070501@163.com; Address: Department of Cardiology, The Third Affiliated Hospital of Inner Mongolia Medical University, Heart Disease and Macrovascular Disease Institute of Baotou City, Baotou, Inner Mongolia, China.
} 
from December 2012 to December 2013, were selected. All patients were fasting overnight for 12 hours. $3 \mathrm{ml}$ blood of the anterior elbow vein was taken from the patient in the morning. The serum Hcy level of all the patients was detected with circulating enzyme method by the laboratory department of our hospital. The results were divided into two groups according to the results of Hcy. A total of 58 cases, including 32 males and 26 females were enrolled in $\mathrm{H}$ type hypertension group as Hcy $\geq 15.4 \mu \mathrm{mol} / \mathrm{L}$, with an average age of $(71.3 \pm 5.1)$ years. And 42 cases were enrolled in pure hypertension group, including 25 males and 17 females, with an average age of $(68.9 \pm 4.5)$ years due to Hcy $<15.4 \mu \mathrm{mol} / \mathrm{L}$. All patients should follow the inclusion criteria: (1) age > 65 years; (2) the patients with hypertension were in accordance with the diagnostic criteria of the Chinese Guidelines for the Prevention and Treatment of Hypertension (2010). Exclusion criteria: secondary hypertension, non-hypertensive heart disease, severe heart failure, diabetes, hyperthyroidism, hypothyroidism, severe liver and renal insufficiency, and recent use of vitamin B and folic acid and other drugs affecting the metabolism of Hcy were also not included.

\subsection{Observational indexes and methods}

Patient history data were collected, including age, gender, hypertension course, smoking and so on. Height and weight were measured by specialists to calculate body mass index (BMI). $4 \mathrm{ml}$ of blood was extracted from the anterior elbow vein blood after fasting $12 \mathrm{~h}$, and serum Hcy was detected by circulating enzyme. Reference range: serum Hcy 0.0-15.4 $\mu \mathrm{mol} / \mathrm{L}$, colorimetric determination of fasting blood glucose (FPG), total cholesterol (TC), triglyceride (TG), low-density lipoprotein-cholesterol (LDL-C), high-density lipoproteincholesterol (HDL-C) from all patients were performed by the HITACHI7600-020 automatic biochemical analyzer.
Model MOBIL-O-GRAPH, an ambulatory blood pressure monitoring system made by German IEM company, was used in the study and it was a noninvasive portable and cuffless blood pressure monitor. Quantitative indexes including BPV $24 \mathrm{~h}$ systolic blood pressure standard deviation (24hSSD), daytime systolic blood pressure standard deviation (dSSD), nighttime systolic blood pressure standard deviation (nSSD), $24 \mathrm{~h}$ diastolic blood pressure standard deviation (24hDSD), day diastolic blood pressure standard deviation (dDSD), nocturnal diastolic blood pressure standard deviation (nDSD), the above data were available by the $24 \mathrm{hABPM}$ instrument. In this study, $24 \mathrm{hSSD}$, dSSD and $\mathrm{nSSD}$ were used as quantitative indexes of systolic and pressure variability (SBPV).

\subsection{Statistical methods}

The data were analyzed by SPSS11.0 software package. The measurement data were expressed as mean \pm standard deviation $(\bar{x} \pm \mathrm{s})$. The $\chi^{2}$ test and $t$-test were adopted on comparison among groups. Pearson correlation analysis was used to analyze the quantitative indexes. Multivariate linear regression was used in multivariate analysis, and variance analysis was used in regression model hypothesis test. $P<.05$ was considered statistically significant.

\section{Results}

\subsection{Comparison of general clinical data between the two groups}

There were no significant differences in age, sex, hypertension course, smoking, BMI, FBG and blood lipid between $\mathrm{H}$ type hypertension group and pure hypertension group ( $p$ $>.05$, see Table 1 ).

Table 1: Comparison of general clinical data between the two groups

\begin{tabular}{|c|c|c|c|c|}
\hline Project & Pure hypertension group (42 cases) & H type hypertension group ( 58 cases) & $t / \chi^{2}$ & $p$ \\
\hline Age (years) & $68.9 \pm 4.5$ & $71.3 \pm 5.1$ & 0.142 & .138 \\
\hline Male/Female (cases) & $25 / 17$ & $32 / 26$ & 3.164 & .074 \\
\hline Hypertension course (years) & $11.55 \pm 4.01$ & $13.26 \pm 4.62$ & 0.246 & .128 \\
\hline Smoking (n, \%) & $17(40.5)$ & $36(62.09)$ & 2.187 & .185 \\
\hline BMI $\left(\mathrm{kg} / \mathrm{m}^{2}\right)$ & $23.54 \pm 2.78$ & $24.00 \pm 2.63$ & 0.213 & .133 \\
\hline $\mathrm{GLU}(\mathrm{mmol} / \mathrm{L})$ & $5.49 \pm 1.12$ & $5.93 \pm 1.37$ & 0.224 & .127 \\
\hline $\mathrm{TG}(\mathrm{mmol} / \mathrm{L})$ & $1.70 \pm 0.41$ & $1.81 \pm 0.38$ & 1.864 & .065 \\
\hline $\mathrm{TC}(\mathrm{mmol} / \mathrm{L})$ & $4.23 \pm 1.03$ & $4.77 \pm 1.26$ & 1.718 & .074 \\
\hline HDL-C (mmol/L) & $1.34 \pm 0.44$ & $1.25 \pm 0.32$ & 0.545 & .578 \\
\hline LDL-C (mmol/L) & $2.71 \pm 0.63$ & $2.86 \pm 0.76$ & 1.934 & .061 \\
\hline
\end{tabular}

Note. BMI: body mass index; HDL-C: high-density lipoprotein-cholesterol; LDL-C: low-density lipoprotein-cholesterol 
Table 2: Comparison of BPV between the two indexes $(\bar{x} \pm \mathrm{s})$

\begin{tabular}{lllll}
\hline Project & Pure hypertension group (42 cases) & H type hypertension group (58 cases) & $\boldsymbol{t}$ & $\boldsymbol{p}$ \\
\hline 24HSSD & $12.80 \pm 2.23$ & $16.01 \pm 3.11^{\triangle \Delta}$ & 7.27 & .003 \\
dSSD & $11.58 \pm 2.30$ & $13.86 \pm 2.26^{\triangle}$ & 3.814 & .039 \\
nSSD & $9.41 \pm 1.65$ & $11.71 \pm 2.34^{\triangle}$ & 3.926 & .034 \\
24hDSD & $9.67 \pm 1.91$ & $10.27 \pm 1.70$ & 0.285 & .612 \\
dDSD & $9.19 \pm 1.48$ & $9.68 \pm 1.82$ & 0.259 & .693 \\
nDSD & $7.92 \pm 1.35$ & $9.10 \pm 1.63$ & 0.391 & .512 \\
\hline
\end{tabular}

Note. 24hDSD: standard deviation of 24h diastolic blood pressure; dSSD: standard deviation of daytime systolic blood pressure; nSSD: standard deviation of nighttime systolic blood pressure; dDSD: standard deviation of daytime diastolic blood pressure; nDSD: standard deviation of nighttime diastolic blood pressure. Compared with pure hypertension group, ${ }^{\Delta} p<.05, \Delta \Delta p<.01$

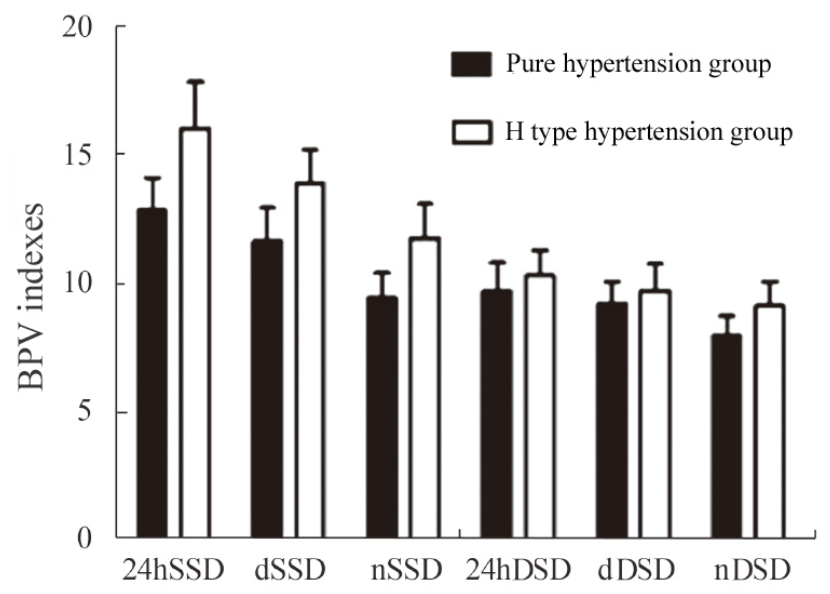

Figure 1: Comparison of BPV between $\mathrm{H}$ type hypertension and pure hypertension

\subsection{Comparison of BPV test between the two groups}

The levels of 24hSSD, dSSD and nSSD were higher in patients with $\mathrm{H}$ type hypertension than those in patients with hypertension alone $(p<.05$ or $p<.01)$. There was no signif- icant difference in 24hDSD, dDSD and $\mathrm{nDSD}$ between the two groups $(p>.05$, see Table 2 and Figure 1$)$.

\subsection{Correlation between 24hSSD, dSSD, nSSD and the indexes}

Pearson correlation analysis was used to analyze the correlation between gender, age, hypertension course, smoking, BMI, FBG, Hcy, TG, TC, HDL-C, LDL-C with 24hSSD, dSSD and nSSD. Results: There were significant positive correlations between Hcy, TC, LDL-C and 24hSSD, dSSD, nSSD $(r=0.201-0.508, p<.05$ or $p<.01$, see Table 3$)$.

\subsection{Multiple regression analysis between 24hSSD, dSSD, nSSD and the indexes}

24hSSD, dSSD, nSSD were taken as dependent variables, age, hypertension course, smoking, sex, Hcy, LDL-C, TC, TG, HDL-C and BMI as independent variables for multiple linear regression analysis, whose results suggested that Hcy and TC were the influence factors of $24 \mathrm{hSSD}$, dSSD and $\mathrm{nSSD}(\mathrm{B}=0.194-0.546, p<.05$ or $p<.01$, see Tables $4-6)$.

Table 3: Pearson correlation analysis between 24hSSD, dSSD, nSSD and the indexes

\begin{tabular}{|c|c|c|c|c|c|c|}
\hline \multirow{2}{*}{ Project } & \multicolumn{2}{|c|}{ 24hSSD } & \multicolumn{2}{|c|}{ dSSD } & \multicolumn{2}{|c|}{ nSSD } \\
\hline & $\boldsymbol{r}$ & $p$ & $\boldsymbol{r}$ & $p$ & $r$ & $p$ \\
\hline Male/Female & 0.035 & .731 & 0.008 & .939 & 0.072 & .479 \\
\hline Age & 0.154 & .129 & 0.131 & .195 & 0.133 & .188 \\
\hline Hypertension course & 0.032 & .897 & 0.010 & .939 & 0.086 & .399 \\
\hline Smoking & 0.152 & .134 & 0.171 & .091 & 0.143 & .158 \\
\hline BMI & 0.062 & .056 & 0.051 & .641 & 0.079 & .438 \\
\hline GLU & 0.044 & .066 & 0.064 & .529 & 0.005 & .958 \\
\hline TG & 0.057 & .731 & 0.149 & .142 & 0.166 & .101 \\
\hline $\mathrm{TC}$ & 0.288 & .004 & 0.256 & .012 & 0.212 & .035 \\
\hline LDL-C & 0.255 & .011 & 0.232 & .014 & 0.201 & .047 \\
\hline HDL-C & -0.173 & .089 & -0.136 & .180 & -0.180 & .075 \\
\hline Hcy & 0.406 & .001 & 0.508 & .001 & 0.343 & .001 \\
\hline
\end{tabular}

Note. BMI: body mass index; HDL-C: high-density lipoprotein-cholesterol; LDL-C: low-density lipoprotein-cholesterol; Hcy: homocysteine 
Table 4: Multiple regression analysis of 24hSSD and the indexes

\begin{tabular}{lllllll}
\hline Project & B & SPRC & $\mathbf{9 5 \%}$ CI & $\boldsymbol{S E}$ & $\boldsymbol{t}$ & $\boldsymbol{p}$ \\
\hline Constant & -7.137 & - & $-16.925-0.256$ & 4.925 & -1.449 & .151 \\
Hcy & 0.305 & 0.395 & $0.175-0.435$ & 0.065 & 4.674 & .001 \\
TC & 0.546 & 0.205 & $0.055-1.037$ & 0.247 & 2.209 & .032 \\
\hline
\end{tabular}

Table 5: Multiple regression analysis of dSSD and the indexes

\begin{tabular}{lllllll}
\hline Project & B & SPRC & 95\% CI & SE & $\boldsymbol{t}$ & $\boldsymbol{p}$ \\
\hline Constant & -2.734 & - & $-11.075-5.607$ & 2.461 & -0.6510 & .571 \\
Hcy & 0.340 & 0.513 & $0.227-0.454$ & 0.057 & 5.964 & .001 \\
TC & 0.429 & 0.187 & $0.003-0.854$ & 0.216 & 2.004 & .047 \\
\hline
\end{tabular}

Table 6: Multiple regression analysis of nSSD and the indexes

\begin{tabular}{lllllll}
\hline Project & B & SPRC & 95\% CI & $\boldsymbol{S E}$ & $\boldsymbol{t}$ & $\boldsymbol{p}$ \\
\hline Constant & 0.522 & - & $-7.474-8.571$ & 4.023 & 0.130 & .897 \\
Hcy & 0.194 & 0.248 & $0.250-0.426$ & 0.199 & 2.356 & .020 \\
TC & 0.468 & 0.344 & $0.088-0.302$ & 0.053 & 3.651 & .001 \\
\hline
\end{tabular}

\section{Discussion}

BPV refers to the fluctuation of blood pressure over a period of time. It is a normal physiological phenomenon that systolic blood pressure and diastolic blood pressure of healthy people have a fluctuation range of $25 \%$ in 24 hours. ${ }^{[1]}$ The risk of vascular disease due to the synergistic effect of high Hcy and hypertension is much higher than that of high Hcy in combination with other risk factors. ${ }^{[2]}$ Recently, a large prospective study in China has confirmed that the risk of cardiovascular and cerebrovascular events in patients with $\mathrm{H}$ type hypertension is 8.5 times and 3.9 times respectively higher than those in patients with hypertension or Hcy. ${ }^{[3]}$

By comparing the BPV indexes of two groups of patients, it was found that 24hSSD, dSSD and nSSD in H type hypertension group were significantly higher than those in pure hypertension group (all $p<.05$ or $p<.01$ ). There was no significant difference between 24hDSD, dDSD and nDSD of the two groups (all $p>.05$ ), which was consistent with the research reports of Xu BL. ${ }^{[4]}$ This study suggested that Hcy in elderly patients with $\mathrm{H}$ typertension and SBPV were closely related, indirectly reflecting that the prognosis of elderly patients with $\mathrm{H}$ type hypertension was worse than that of patients with pure hypertension. It provides a basis for $\mathrm{H}$ type hypertension patients who are prone to have cardiovascular and cerebrovascular diseases than those with pure hypertension.

The study found that there was a significant difference of SBPV between the two groups, but no difference of DBPV between the two groups. The reason may be that the el- derly may have different degrees of organ degenerative diseases, and a variety of blood pressure regulation mechanism in the body is unbalance with the increase of age. It is mainly manifested as neuro-hormonal regulation, sympathetic neural activity regulation and decreased baroreflex sensitivity. ${ }^{[5-8]}$ In addition, the fact that the decreased arterial elasticity and the increased pulse pressure of the elderly result in the increase of simple systolic pressure, a characteristic in patients with $\mathrm{H}$ type hypertension.

Hypertension and high Hcy are the two major elements of hypertension and they are not a simple combination of two factors. High Hcy can promote the occurrence and development of hypertension. That's the reason why SBPV is higher in patients with $\mathrm{H}$ type hypertension than that with pure hypertension. In this study, the Pearson correlation analysis showed that the level of SBPV in the elderly patients with $\mathrm{H}$ type hypertension was positively correlated with the level of serum Hcy. The multiple linear regression analysis further confirmed that Hcy was the influencing factor of SBPV in elderly patients with $\mathrm{H}$ type hypertension, and the serum Hcy was closely related to SBPV. Self oxidation of Hcy causes vascular endothelial cell injury, and the change of vascular shear stress leads to abnormal vascular structure and vascular compliance. Plus, Hcy leads to injury of vascular endothelial cells induced by excessive active oxygen in the body, resulting in the renin-angiotensin system activation induced by sympathetic nerve and vagus nerve on vascular regulation imbalance, and stimulates BPV. ${ }^{[9]}$ In addition, oxidative stress affects the biological activity of nitric oxide, causes the damage of arterial endothelium, 
leads to the change of vascular structure and function, and the increase of intimal medial thickness. Arterial stiffness increases and compliance decreases, resulting in increased BPV and atherosclerosis. ${ }^{[10,11]}$

At present, the exact relationship between Hcy and BPV in elderly patients with hypertension is still not very clear. However, from the results of this study, serum Hcy level was closely related to SBPV in elderly patients with hypertension, and was the influencing factor of SBPV, suggesting that serum Hcy may be involved in the occurrence and development of elderly hypertension. Thus, BPV was higher in patients with $\mathrm{H}$ type hypertension than that with pure hypertension. In the future, relevant investigations should be carried out at all levels to provide an effective theoretical basis for the diagnosis and treatment of hypertension. In addition, the study also confirmed the positive correlation between BPV and LDL-C in patients with hypertension through Pearson, which is consistent with a domestic study about the relationship between low density lipoprotein and BPV ${ }^{[12]}$ There was a significant positive correlation between LDL-C and BPV, probably due to: (1) The release of bradykinin, an agent that inhibits endothelium-dependent vasodilatation by ox-LDL, promotes vasoconstriction and causes the increase of SBPV; (2) The high degree of cytotoxicity of ox-LDL leads to the thickening of the intima, which results in the decrease of the compliance of the arterial wall and increase of BPV. ${ }^{[13]}$ This study further found LDL-C did not enter the regression equation by multivariate linear regression analysis, suggesting that SBPV is not an influencing factor. Whether it is due to the limitation of the sample size of this study remains to be discovered. This study found that there was a significant positive correlation between SBPV and TC in hypertensive patients, and TC was the influencing factor of SBPV, which was consistent with

\section{References}

[1] Parati G, Pomidossi G, Albini F, et al. Relationship of 24 hour blood pressure mean and variability to severity of target-organ damage in hypertension. J Hypertens. 1987; 5(1): 93-98. https ://doi .org/ 10.1097/00004872-198702000-00013

[2] Towfighi A, Markovic D, Ovbiagele B. Pronounced association of elevated serum homocysteine with stroke in subgroups of individuals: a nationwide study. J Neurol Sci. 2010; 298(1-2): 153-157. PMid: 20810133. https://doi.org/10.1016/j.jns . 2010.07.013

[3] Graham IM, Daly LE, Refsum HM, et al. Plasma homocysteine a risk factor for vascular disease. The European Concerted Action Project. JAMA. 1997; 277(22): 1775-1781. https://doi.org/ $10.1001 /$ jama. 1997.03540460039030

[4] Xu BL, Ma LN, Yang Wei. The effect of serum homocysteine level on blood pressure variability in elderly patients with hypertension. Journal of Shanxi Medical University. 2013; 44(11): 872-874.

[5] Kaplan NM. Kaplan's Cinical Hypertension, $9^{\text {th }}$ edition (2005). Can J Cardiol. 2007; 23(7): 605.

[6] Rothwell PM, Howard SC, Dolan E, et al. Effects of beta blockers and calcium-channel blockers on within-individual variability in the above findings. The significant positive correlation between TC, LDL-C, Hcy and SBPV in elder patients with hypertension was also shown in our study. Among them, TC and Hcy were the influencing factors of SBPV, suggesting that dyslipidemia and high Hcy both affected SBPV in elderly patients with hypertension. Both dyslipidemia and high Hcy have been identified as one of the major risk factors for atherosclerosis. Zhang $\mathrm{MH}$ et al. ${ }^{[14]}$ reported that the cervical-femoral pulse wave velocity was significantly higher in patients with $\mathrm{H}$ type hypertension than that in patients with pure hypertension. Therefore, the variability of BPV especially systolic blood pressure is closely related to vascular sclerosis.

This study showed that SBPV in elderly patients with $\mathrm{H}$ type hypertension was significantly higher than that in patients with pure hypertension. Among them, high Hcy is closely related to SBPV, which is the influencing factor of SBPV. It indicates that elderly patients with $\mathrm{H}$ type hypertension are more likely to have target organ damage than those with pure hypertension. Comprehensive considerations are required for the clinical staff to treat patients with $\mathrm{H}$ type hypertension. On one hand, the control of the blood pressure level should be taken into account by reducing the high Hcy for this kind of patients. On the other hand, we reduce the activity of sympathetic nerve on the basis of improving the autonomic nerve function of cardiovascular system, thereby reducing BPV in hypertensive patients, and further reduce the occurrence of target organ damage in hypertensive patients.

\section{Conflicts of Interest Disclosure}

The authors have no conflicts of interest related to this article.

blood pressure and risk of stroke. Lancet Neurol. 2010; 9(5): 469480. https://doi .org/10.1016/S1474-4422(10)70066-1

[7] Webb AJ, Fischeru, Mehta Z, et al. Effects of antihypertensive drug class on interindividual variation in blood pressure and risk of stroke: a systematic review and meta-analysis. Lancet. 2010; 375(9718): 906-915. https ://doi .org/10.1016/ S0140-6736 (10)60235-8

[8] Chen PP, Huang JF. Research Progress on 24 hour blood pressure fluctuation. Chinese Circulation Journal. 2011; 26(2): 156-158.

[9] Ye P. Hypertension complicated with dyslipidemia: we need a twopronged strategy. Chinese Community Doctors. 2010(34): 8.

[10] Na KX, Yu Ping, Zhang GY. Attention should be paid to the relationship between homocysteine and hypertension. Chinese Journal for Clinicians. 2010; 38(1): 3-6.

[11] Sen U, Moshal KS, Tyagi N, et al. Homocysteine induced myofibroblast differentiation in mouse aortic endothelial cells. J Cell Physiol. 2006; 209(3): 767-774. PMid: 16972260. https://doi. org/10.1002/jcp. 20752

[12] Ye Z, Ye Y. The relationship between low density lipoprotein and blood pressure variability in 50 patients with hypertension. Journal of Hainan Medical University. 2011; 17(4): 482-483. 
[13] Elmouchi DA, Bates ER. Platelet glycoprotein IIb/IIIa inhibitor therapy in non-ST segment elevation acute coronary syndromes. Minerva Cardioangiol. 2003; 51(5): 547-560. PMid: 14551523.
[14] Zhang MH, Ye P, Luo LM, et al. Community investigation of type $\mathrm{H}$ hypertension. Chinese Journal of Cardiovascular Rehabilitation Medicine. 2012; 21(5): 482-486. 